\title{
Invasibility in a spatiotemporally fluctuating environment is determined by the periodicity of fluctuations and resident turnover rates
}

\author{
Donald R. Schoolmaster Jr* and Robin E. Snyder \\ Department of Biology, Case Western Reserve University, 10900 Euclid Avenue, Cleveland, OH 44106-7080, USA
}

\begin{abstract}
The ability of a species to invade a community is influenced by the traits of the invader, the resident community and the environment. However, qualitative generalizations are possible. Using a model of perennial plants in a spatiotemporally fluctuating environment, we find that fluctuating environments may be more or less invasible than static environments. Invasibility is strongly dependent on the interaction of the difference in turnover rates of resident and invader populations and the rate of temporal change of the environment. If resident population turnover is faster than the invader's, then invasibility is an initially positive, decreasing function of the period temporal variation, such that invasibility is increased by rapid temporal fluctuations but slightly reduced in slowly fluctuating environments. If resident turnover is slower than the invader's, then invasibility is an initially negative, increasing function of temporal period, such that invasibility is reduced in rapidly changing environment facilitated by slow temporal fluctuations. These results are explained by the relative abilities of resident and invader populations to successfully respond to environmental variation at different temporal scales.
\end{abstract}

Keywords: invasibility; environmental variation; spatiotemporal; germination; turnover

\section{INTRODUCTION}

The concept of community invasibility has become central to the study of species invasions (Lodge 1993; Burke \& Grime 1996; Tilman 1997; Lonsdale 1999; Davis et al. 2005). Invasibility is an emergent property of the community which is determined by the interaction of traits of the environment (Alpert et al. 2000), the resident community (e.g. Crawley 1987) and the putative invader (e.g. Rejmanek \& Richardson 1996). Recently, a conceptual connection has been made that the processes which influence coexistence and diversity among resident species are the same as those that promote invasion of non-resident species (Stohlgren et al. 1999), implying that the community ecology theories used to study coexistence may also be used to understand species invasion (Huston 1994; D'Antonio \& Levine 1999; Davis et al. 2000; Shea \& Chesson 2002; Huston 2004).

Environmental variation, which has been shown theoretically to contribute to robust coexistence among competing plants (Chesson 2000a; Snyder \& Chesson 2004; Roxburgh et al. 2004), has, through a different line of reasoning, been suggested as a general mechanism of invasibility (Davis et al. 2000). The fluctuating resource hypothesis of invasibility suggested by Davis et al. (2000) states that fluctuation in resource supply reduces competition intensity from resident vegetation, resulting in periods of increased invasibility. This hypothesis predicts that communities will be more susceptible to invasion when there is an increase in the amount of unused resources. Increased resource availability could result from

\footnotetext{
*Author for correspondence (drs34@case.edu).
}

Electronic supplementary material is available at http://dx.doi.org/10. 1098/rspb.2007.0118 or via http://www.journals.royalsoc.ac.uk. a decrease in uptake rates of the resident vegetation, as would result from certain kinds of disturbance or from increased supply rates.

Recent empirical evidence supporting (Davis \& Pelsor 2001; Foster \& Dickson 2004) and refuting (Gilbert \& Lechowicz 2005; Walker et al. 2005) the fluctuating resource hypothesis have been reported. Viewed in light of theoretical work on coexistence in fluctuating environments, the variable outcomes among these studies are not surprising. For example, Chesson (2000a) explains how in temporally variable environments, fluctuation-dependent coexistence mechanisms may increase or decrease the long-run lowdensity growth rate of an invading species, and Snyder \& Chesson (2004) show, using a model of competition among annual plants in a spatially heterogeneous environment, that the strength of coexistence depends upon the spatial scale of environmental variation and the spatial scales over which the resident and invading species interact with the environment (through dispersal) and one another (through competition). These studies suggest that understanding how environmental variability in space and/or time may contribute to the invasibility of a plant community requires information on the nature of the fluctuations in the environment, and the abilities of the resident and invading species to respond to environmental variability.

In this paper, we describe and analyse a model of plant competition in a spatiotemporally variable environment. We use this model to understand how the spatial and temporal scale of environmental variation interacts with the life-history traits of the resident species to determine the invasibility of plant communities. We find that environmental fluctuations may increase or decrease the invasibility of a community. We also find that the turnover rate of individuals of the resident species strongly interacts 
with the temporal scale of environmental variation to determine invasibility, and that the patterns of invasibility determined by these traits are consistent over a wide range of other ecological characteristics of both the resident and the invading species.

\section{MODEL}

We use an extension of the plant model in Snyder \& Chesson (2004), adding adult survivorship. In the current model, seedling germination/establishment probability varies in space and time. Adults produce seeds that are passively dispersed. Seeds germinate to compete with nearby adults for resources, with increased competition reducing the proportion of germinated seeds that become established.

We make a mathematical formulation of this verbal description in the following way. An adult plant of species $j$ survives with probability $\left(1-\delta_{j}\right)$. Surviving adults produce $F_{j}$ seeds which disperse a distance $z$ away from the parent plant with probability $k_{j}(z)$, where $k_{j}(z)$ is the dispersal kernel. This means that the number of seeds arriving at location $x$ at time $t+1$ is equal to the number of seeds produced at location $y$ at time $t, F_{j} n_{j}(y, t)$, times the probability of dispersing from $y$ to $x, k_{j}(x-y)$, summed over all $y$, yielding

$\int_{-\infty}^{\infty} k_{j}(x-y) F_{j} n_{j}(y, t) \mathrm{d} y$.

(For simplicity, we assume that space is one-dimensional; however, results in two-dimensional space should not be qualitatively different.) A seed at location $x$ and time $t$ germinates and becomes established with probability $E_{j}(x, t)$ in the absence of neighbours.

Competition, which reduces the probability of establishment, depends on a weighted average of the local density of adults: $\int_{-\infty}^{\infty} U_{j k}(x-y) n_{k}(y, t) \mathrm{d} y$. Thus, in the presence of neighbours, the probability that a seed becomes established is $E_{j}(x, t) / \int_{-\infty}^{\infty} U_{j k}(x-y) n_{k}(y, t) \mathrm{d} y$. The weight function or 'competition kernel', $U_{j k}(z)$, declines with distance $z$, so that more distant competitors affect establishment to a lesser extent. The characteristic width of $U_{j k}(z)$ can be thought of as the radius of competition. The total contribution of species $k$ to the competition experienced by species $j$ is the number of adults at location $y, n_{k}(y, t)$, times the weight function for distance $x-y, U_{j k}(x-y)$, summed over all $y$ : $\int_{-\infty}^{\infty} U_{j k}(x-y) n_{k}(y, t) \mathrm{d} y$. Normally, we would sum over competitor species $k$ to get the full competition experienced by species $j$; however, we assume that there is a 'resident' species at stationary density that contributes significantly to the competition experienced by potential invaders. The assumption of a single resident species was made to benefit analytical tractability, but is not unreasonable. Crawley et al. (1999) found that the invasibility of experiment plots was most strongly influenced by the presence of the competitively dominant species in the system.

In the presence of competition, the number of species $j$ individuals that become established at $x$ at the end of the year $t$ is

$\frac{E_{j}(x, t)}{\int_{-\infty}^{\infty} U_{j k}\left(x-y_{1}\right) n_{k}\left(y_{1}, t\right) \mathrm{d} y_{1}}\left(\int_{-\infty}^{\infty} k_{j}(x-y) F_{j} n_{j}(y, t) \mathrm{d} y\right)$.
We can rewrite this as $\lambda_{1 j}(x, t) n_{j}(x, t)$, where $\lambda_{1 j}(x, t)$ is given by

$\frac{E_{j}(x, t) \int_{-\infty}^{\infty} k_{j}(x-y) F_{j} n_{j}(y, t) \mathrm{d} y}{n_{j}(x, t) \int_{-\infty}^{\infty} U_{j k}\left(x-y_{1}\right) n_{k}\left(y_{1}, t\right) \mathrm{d} y_{1}}$.

Thus, the local per capita growth rate of species $j$ at site $x$ at time $t$ is equal to the growth to recruitment $\lambda_{1 j}(x, t)$ plus adult survivorship $\lambda_{2 j}: \lambda_{j}(x, t)=\lambda_{1 j}(x, t)+\lambda_{2 j}$, where $\lambda_{2 j}=1-\delta_{j}$. For the figures in this paper, we use exponentially declining forms for the dispersal and competition kernels: $k_{j}(x)=1 /\left(2 a_{j}\right) \mathrm{e}^{-|x| / a_{j}}$ and $U_{j k}(x)=1 /\left(2 b_{j k}\right) \mathrm{e}^{-|x| / b_{j k}}$. The parameters $a_{j}$ and $b_{j k}$ define characteristic widths for the kernels, such that $a_{j}$ is the average dispersal distance of species $j$ and $b_{j k}$ is the average scale of competition between species $j$ and $k$. We used $b_{j k}=b_{k k}=1$ and $a_{\mathrm{r}}=a_{\mathrm{i}}=1$ for the figures in this paper.

We assume that both species experience favourable conditions in the same places at the same time and let the establishment probabilities of both species vary sinusoidally in space and time

$E_{j}(x, t)=c \sin \left(\frac{2 \pi}{L} x\right) \sin \left(\frac{2 \pi}{T} t\right)+\bar{E}$

where $c$ is a small parameter. The form of equation (2.4), while simplistic, is a useful one to explore because by using Fourier analysis, any spatiotemporal pattern can be built as a sum of products of sinusoids at different spatial and temporal scales (Nisbet \& Gurney 1982; Edelstein-Keshet 2005). Using the form in equation (2.4), which represents small sinusoidal fluctuations about the average establishment rate, allows us to explore invasibility as a function of fluctuations at different spatial $(L)$ and temporal $(T)$ periods. The scale of $L$ can be thought of in terms of units relative to the size of an individual plant. For example, $L=50$ indicates that in a given season, consecutive peaks in germination/establishment probability occur 50 plant size units apart. The scale of $T$ can be thought of in terms of growing seasons or years in a seasonal environment. For example, $T=2$ indicates that, in a given area, years of peak germination/establishment occur every other year.

We measure the success of invasion by determining whether an invading species can increase from a regionally low density in the presence of a resident species which has reached a stationary distribution. By regionally, we mean the spatial extent at which the system is effectively closed. This scale has been referred to as both the regional scale (Chesson 2000b) and the metacommunity scale (Leibold et al. 2004). We analyse a two-species system, with the invader denoted by subscript ' $\mathrm{i}$ ' and the resident species by subscript ' $r$ '. Since we are interested in the role of environmental heterogeneity on invasibility, parameters are set up such that in the absence of environmental heterogeneity, the long-run regional growth rate of the invader is zero (i.e. the invader just barely fails to invade). Invasion is successful if the invading species has a positive long-run regional growth rate, $\bar{r}_{\mathrm{i}}$, when it is at low density. The larger $\bar{r}_{\mathrm{i}}$ is, the faster the invader increases from low density and the more robust its persistence. Negative values of $\bar{r}_{\mathrm{i}}$ indicate that environmental variability reduces invasibility. We calculate the long-run regional growth rate as the geometric mean of the regional growth rate: $\overline{r_{\mathrm{i}}}=\left\langle\ln \widetilde{\lambda}_{\mathrm{i}}\right\rangle_{t}$, where the regional scale growth rate at time $t$, $\vec{\lambda}_{\mathrm{i}}(t)$, is defined as the regional density at time $t+1$ divided 


$$
\text { (a) }
$$

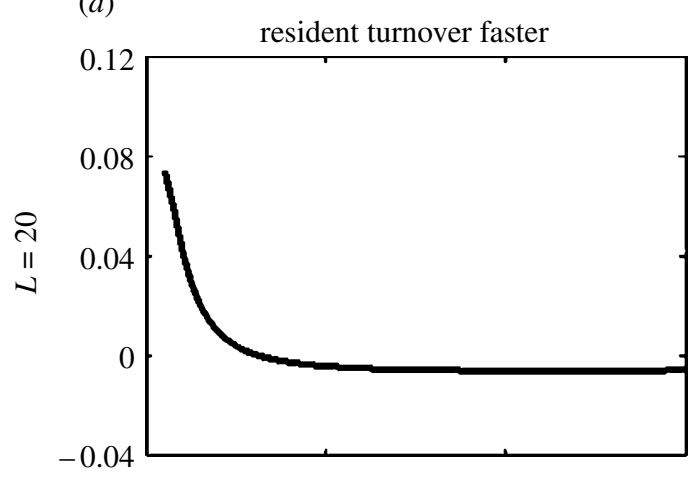

(b)

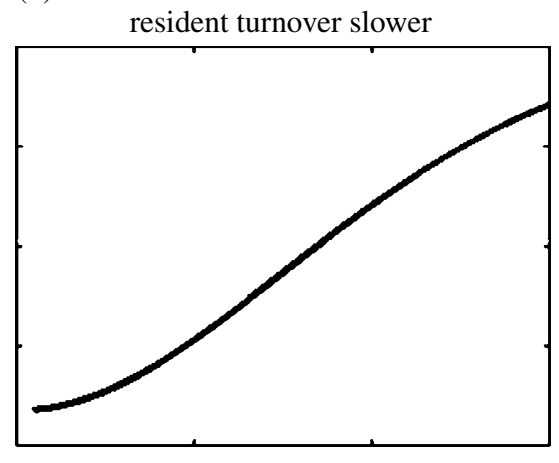

$1:-$

$(c)$

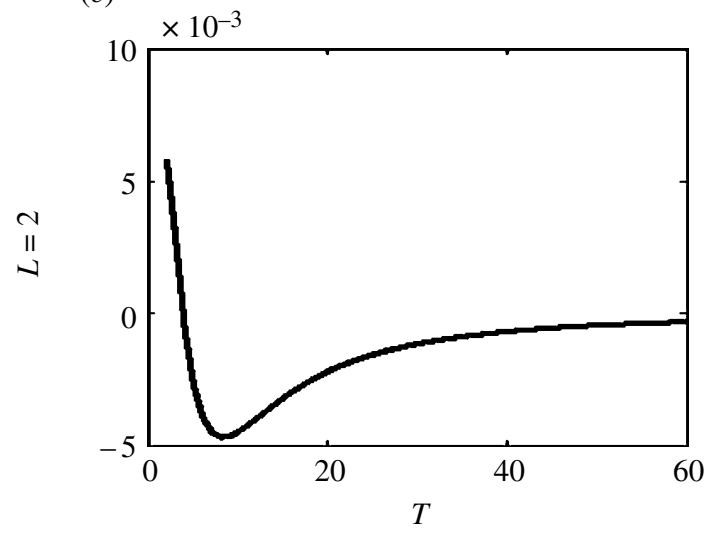

(d)

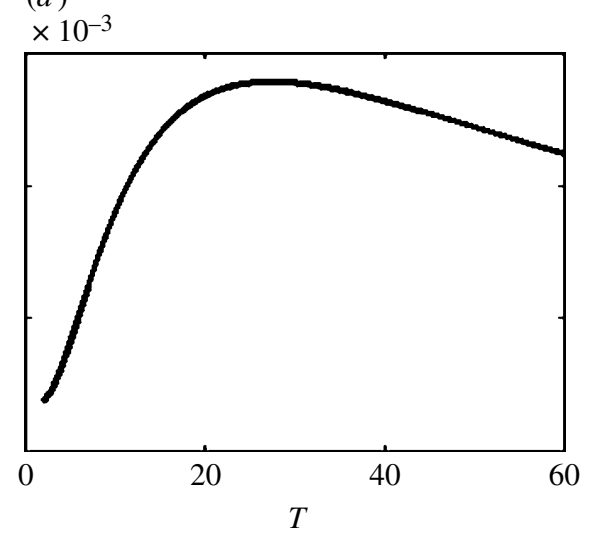

Figure 1. The long-run regional growth rate of the invader $\left(\bar{r}_{\mathrm{i}}\right)$ versus temporal period $(T)$ at different spatial $(L)$ periods. If resident turnover is faster than the invader's $\left((a, c) F_{\mathrm{r}}=9.0, \delta_{\mathrm{r}}=0.90, F_{\mathrm{i}}=5.0, \delta_{\mathrm{i}}=0.5\right)$, then opportunities exist for invader in rapidly fluctuating environments. However, if resident turnover is slower than the invader's $\left((b, d) F_{\mathrm{r}}=1.0, \delta_{\mathrm{r}}=0.10, F_{\mathrm{i}}=5.0\right.$, $\left.\delta_{\mathrm{i}}=0.5\right)$, then invaders experience opportunities in slowly fluctuating environments, while rapid temporal fluctuations increase resistance to invasion. For all curves, the mean establishment $\langle E\rangle_{x, t}=0.5, c=0.4$ and fecundities are adjusted with survivorship according to the relationship $F_{j}=10 \times \delta_{j}$ so that the effects of changing survivorship are not confounded with changes in average densities.

by the regional scale density at time $t: \widetilde{\lambda}_{\mathrm{i}}(t)=\overline{n_{\mathrm{i}}}(t+1) / \overline{n_{\mathrm{i}}}(t)$. The second-order perturbative approach we use to calculate $\bar{r}_{\mathrm{i}}$ is derived in the digital appendix of the electronic supplementary material.

In this paper, we examine the relationship between the turnover rates and invasibility. We define the turnover of individuals as the proportion of the population that is replaced by new individuals annually when at a stationary distribution. Examining turnover rates allow us to investigate the effects of adult survivorship while keeping the potentially confounding effect of density constant. We achieve constant density by keeping the ratio of fecundity, $F_{j}$, to adult mortality, $\delta_{j}$, constant. In general, we would not expect the traits of the resident species to be independent of the period of fluctuations in the environment, but treat them as such here so that we may investigate relationships between species traits, periods of environmental variation and invasibility. Thus, our results may be thought to best apply to systems in which the historical pattern of environmental fluctuation has been recently altered (Sher \& Hyatt 1999).

\section{RESULTS}

The long-run regional growth rate of the invader, $\bar{r}_{\mathrm{i}}$, depends on all the parameters of the model in a complex way (figure 1). However, three qualitative generalities emerge that are as follows: (i) spatiotemporal environmental fluctuations may facilitate or inhibit the invasibility of the community, (ii) opportunities for invaders are determined by the interaction of relative differences in the ability to respond to environmental variation and the temporal period of the fluctuations, and (iii) there is no one-scale of temporal fluctuation that maximizes invasion. Rather, invasibility depends on the attributes of the resident and invader, including the scales of dispersal and competition.

The first point demonstrated by the model is that, if both species experience favourable conditions in the same time at the same places in a spatiotemporally fluctuating environment, but differ in rates of population turnover, then environmental variation may increase or decrease the invasibility of a community. Figure 1 shows the relationship between the temporal period of environmental fluctuations and $\bar{r}_{\mathrm{i}}$ in cases where the resident has a faster (figure $1 a, c$ ) and slower (figure $1 b, d$ ) rate of population turnover than the invader, at two scales of spatial variation. In each situation, there are temporal periods that result in positive and negative values of $\bar{r}_{\mathrm{i}}$ for invaders. Environmental fluctuations may increase invasibility by providing spatial and/or temporal refuges from competition with the resident species. For example, a fluctuating environment may result in 'good spots' where resources are unused by the resident species, allowing invaders to establish. However, if the resident species is effective at responding to variability, by quickly increasing its densities in these good spots, the benefit of a favourable 
(a)

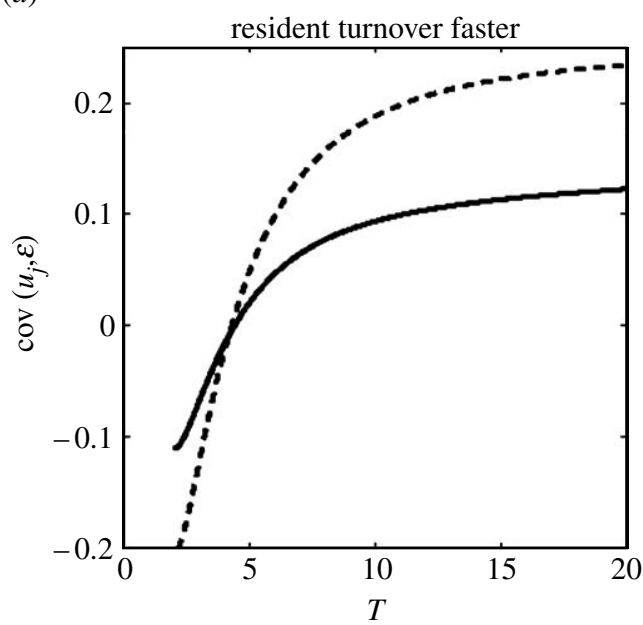

(b)

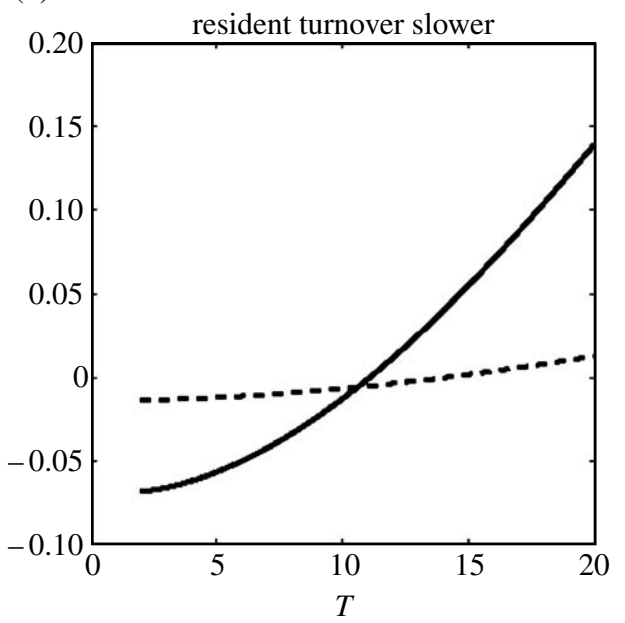

Figure 2. Spatiotemporal covariance of variation in density $u_{j}(x, t)$ and establishment probability $\varepsilon(x, t)$ versus temporal period for resident (dashed) and invader (solid). (a) If resident turnover is faster than the invader's $\left(F_{\mathrm{r}}=9.0, \delta_{\mathrm{r}}=0.90, F_{\mathrm{i}}=5.0\right.$, $\left.\delta_{\mathrm{i}}=0.5\right)$, then the invader population tracks environmental variation less poorly than the resident in rapidly fluctuating environments, but more poorly in slowly fluctuating environments. (b) If resident turnover is slower than the invader's $\left(F_{\mathrm{r}}=1.0\right.$, $\left.\delta_{\mathrm{r}}=0.10, F_{\mathrm{i}}=5.0, \delta_{\mathrm{i}}=0.5\right)$, then the invader population tracks variation more effectively in slowly fluctuating environments. The values $u_{j}(x, t)$ and $\varepsilon(x, t)$ are the zero-centred, normalized variations of $\left\langle n_{j}\right\rangle_{x, t}$ and $\langle E\rangle_{x, t}$, respectively (i.e. $\left.u_{j}(x, t)=n_{j}(x, t) /\left(\left\langle n_{j}\right\rangle_{x, t}\right)-1 ; \varepsilon(x, t)=E(x, t) /\left(\langle E\rangle_{x, t}\right)-1\right)$. Our method to calculate the covariance is presented in the digital appendix of the electronic supplementary material.

environment can be outweighed by the cost of strong competition from a dense local population of residents. As a result, invaders experience a less favourable environment than they would in the absence of fluctuations. Where this occurs, environmental fluctuations work to negate opportunities for invaders from fluctuation-independent sources such as resource partitioning, as discussed for species coexistence by Chesson (2000a). Since the ability of the resident to respond to environmental variability depends on the time-scale of that variation, the period of the environmental fluctuation plays an important role in determining whether fluctuations will result in refuges from or concentration of competition with the resident species.

The relationship between the period of environmental fluctuation and the ability of the resident species to respond and take advantage of those fluctuations suggest the second main point demonstrated by this model: the relationship between $\bar{r}_{\mathrm{i}}$ and the temporal period of fluctuations depends on the difference in population turnover rates between the residents and invaders. If resident turnover is faster than the invader's, the invasibility of communities decreases with increasing temporal period (figure $1 a, c$ ). But, if resident turnover is slower than the invaders, then invasibility increases with increasing temporal period (figure $1 b, d$ ). This phenomenon can be understood by considering the relative abilities of residents and invaders to track changes in the environment. The rate of population turnover affects the ability of the population to respond to environmental variation. Populations with high turnover will tend to track areas that best promoted establishment in recent years. In environments that change rapidly with respect to the growth cycle, areas that are best for establishment in one year are likely to be poorer in the near future. Thus, higher relative resident turnover results in the concentration of resident populations in areas that are poor for establishment, providing opportunities for invaders to experience low competition in areas that are best for establishment.
In terms of the variation-dependent mechanisms described by Chesson (2000b), these opportunities related to the storage effect. In slowly changing environments, in which areas that favour establishment remain for generations, having higher relative turnover allows the resident population to accumulate in areas that are best for establishment, thus providing little opportunity for invaders (figure $2 a$ ). If resident turnover is slower than the invaders, then the resident population averages over rapid environmental variation and is thus less susceptible than the invader in becoming concentrated in areas that are poor for establishment. However, slower turnover also prevents the resident population from effectively tracking longer scale temporal variation as effectively as the invader (figure $2 b, d$ ). In this case, the opportunities provided to invaders are related to positive growth-density covariance (Chesson 2000b).

The scale of spatial variability has an important influence on invasibility. Invasibility generally decreases with decreasing spatial period. This can be understood by considering how the spatial scale of environmental variation affects the competitive environment experienced by the invader. If the scale of spatial variation is large relative to the scale of competition, increasing resident turnover causes the resident population to be clumped, allowing the invader potential spatial refuges from competition. If the scale of spatial variation is short, then the invaders experience a more uniform competitive environment, because they are always close enough to the residents to be affected by them.

The invader's ability to take advantage of recruitment opportunities also depends on its turnover rate. Invaders with low turnover rates are best able to invade rapidly changing environments, whereas those with high turnover rates are best able to invade slowly changing environments (figure 3). As described for the resident, high turnover in rapidly changing environments results in the concentration of the invader population in areas that are not good 
(a)

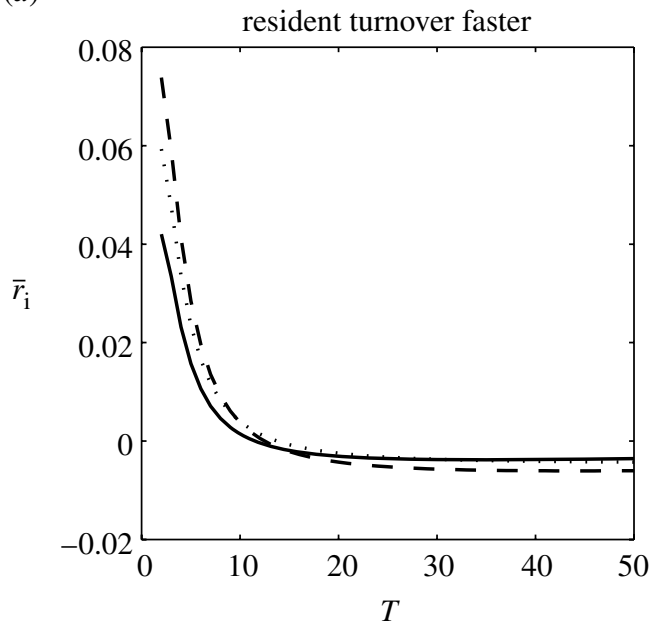

(b)

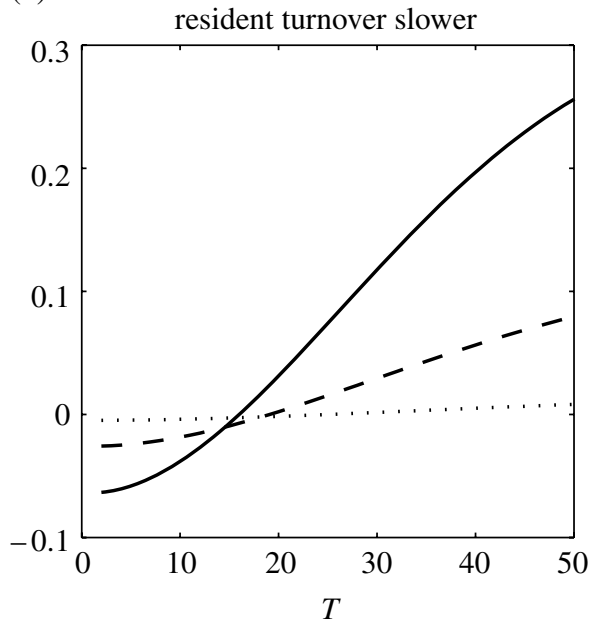

Figure 3. Long-run regional growth rate of the invader versus temporal period for invaders with fast (solid: $F_{\mathrm{i}}=7.5, \delta_{\mathrm{i}}=0.75$ ), medium (dashed: $\left.F_{\mathrm{i}}=5.0, \delta_{\mathrm{i}}=0.50\right)$ and slow (dotted: $\left.F_{\mathrm{i}}=2.5, \delta_{\mathrm{i}}=0.25\right)$ rates of population turnover where $(a)$ the resident has faster turnover than the invader $\left(F_{\mathrm{r}}=9.0, \delta_{\mathrm{r}}=0.9\right)$ and $(b)$ the resident has slower turnover than the invader $\left(F_{\mathrm{r}}=1.0, \delta_{\mathrm{r}}=0.10\right)$.

for establishment. Low turnover prevents the invader from experiencing large responses to the annual changes in the environment, preventing the population from concentrating in areas that are poor for establishment. However, because effective tracking is possible if the environment changes slowly, high invader turnover rates are beneficial for invader populations in slowly changing environments, allowing population accumulation in the areas best for establishment. Low turnover rates prevent the invader from effectively tracking the changing environment, requiring populations to experience both good and poor establishment environments, which results in lowering the regional population growth rate.

The final point suggested by this model is that over the range of possible resident turnover rates, there is not one environment, as characterized by periods of spatial and temporal fluctuation, that is always most invasible. The environment that is most invasible depends on the relative abilities of the residents and invader to respond to the variation. Resident turnover rates that promote invasibility in one temporal environment inhibit invasibility in the other. For example, lower relative resident turnover provides the invader with establishment opportunities in slowly changing environments, but inhibit invasibility in rapidly changing environments. Higher relative resident turnover provides opportunities for invaders in rapidly changing environments, but inhibits invasibility in slowly changing environments.

\section{DISCUSSION}

The fluctuating resource hypothesis of Davis et al. (2000) has intuitive appeal and has been useful in understanding the results of invasion experiments (i.e. Burke \& Grime 1996; Davis \& Pelsor 2001; Foster \& Dickson 2004). We have built upon this work by using a model to understand how spatiotemporal environmental variability interacts with the traits of resident species to influence invasibility. For example, the fluctuating resource hypothesis argues that fluctuation in resource supply affects competition intensity from resident vegetation, resulting in periods of increased invasibility (Davis et al. 2000; Davis \& Pelsor 2001). The hypothesis emphasizes that '...changes in invasibility are often due to changes in the competition intensity from resident vegetation, which in turn results from fluctuations in resource availability' (Davis \& Pelsor 2001). Here, we have shown that increased invasibility is indeed one possible outcome of fluctuations in resource availability. However, we have also shown that environmental fluctuation may concentrate resident competition in such a way as to reduce invasibility. Whether fluctuations increase or decrease, invasibility depends on the interaction of the period of the fluctuations and the ability of the resident and invader populations to respond to them.

Our results are consistent with the findings of Shea \& Chesson (2002), who state that in order for environmental variation to provide opportunities for invaders, the invaders must have an advantage over residents in some places or times via different environmental responses, or via differences in a life-history trait that affects their abilities to respond to the environment. Here, we find that turnover rate, which is related to mean lifespan, is an important life-history trait affecting the ability of populations to respond to spatiotemporal variation. As such, differences in this trait between residents and invaders can provide opportunities for invaders even if both species have similar germination responses.

Since our model calculates invasibility as a function of species traits, the results are potentially useful for understanding how different scales of temporal variation may facilitate invasion across different systems. For example, when controlled for differences in propagule pressure, our results suggest that in herbaceous systems with high turnover rates, short-period temporal fluctuations will facilitate invasibility most effectively. Invasive species that are relatively long lived will be most successful in these systems. On the other hand, in systems where the turnover rates of individuals are low (e.g. forests), invasibility is predicted to be facilitated much more by longer-period temporal fluctuations. Invaders with higher turnover rates will be most successful in these systems. Testing predictions such as these will be challenging. It requires experimentally separating the influences of fluctuationdependent and fluctuation-independent mechanisms of invasibility, which may interact with one another in a 
constructive or destructive manner. As such, using available survey data to correlate turnover rates and invasion success will not be instructive.

Davis et al. (2000) predict that there should be no relationship between productivity and invasibility. They come to this conclusion based, in part, on the results of Goldberg et al. (1999) who found no relationship between productivity and intensity of competition. However, the turnover rate of trees in the New World tropics has been shown to increase with increasing soil fertility (Phillips et al. 2004) and increasing mean annual precipitation (Condit et al. 2004). In addition, Stephenson \& van Mantgem (2005) found a positive relationship between net primary productivity and the turnover rates of individual trees in forest systems at a wide range of spatial scales. If it is generally true that there is a strong positive relationship between individual turnover rates and productivity, our model suggests that invasibility is related to productivity, although not in a simple fashion and not directly due to competition. Whether productivity is positively or negatively associated with fluctuation-dependent, invasibility will depend upon the temporal period of the fluctuation, as previously described for turnover.

For this paper, we have used sinusoidal spatiotemporal variation to investigate the relationships among the period of environmental fluctuation, individual turnover rates and invasibility. Our use of sinusoidal variation is meant to aid analytical tractability. It allows us to isolate and analyse variation at one scale in an attempt to understand how variation at particular scales interacts with the traits of the species to determine invasibility. However, it has the drawback of potentially producing environments with negative correlation structure, which are unusual in natural systems (but see Levine \& Rees 2004). Actual environmental variation can be thought of as being composed of sinusoidal variation at a number of different periods (or frequencies). Recently, various kinds of temperature (Cyr \& Cyr 2003; Vasseur \& Yodzis 2004) and precipitation (Vasseur \& Yodzis 2004) time-series data have been analysed to determine the contribution of variation at different frequencies to the total environmental variation (called the 'colour' of the variation). These studies find that environmental variables often vary more at larger temporal scales than they do at smaller temporal scales. Incorporating this kind of multi-scale environmental variation, which imposes a strictly positive correlation structure, into our model did not affect the relationship between invasibility and turnover (results not shown). In addition, we have found similar relationships between invasibility and turnover in a model of annual plants with a seedbank, suggesting that our results do not depend qualitatively on our choice of model.

Understanding the role of environmental variation in the assembly and maintenance of communities is vital to our understanding of the structure of natural communities. It is also necessary to our ability to predict the ecological effects of changing patterns of environmental variability. Theoretical models show that the colour of environmental noise can have important implications for the dynamics and persistence of populations (Kaitala et al. 1997; Ripa et al. 1998; Cuddingtion \& Yodzis 1999). Our model demonstrates that the colour of environmental noise can also have important effects on the invasibility of communities. In addition, this model provides insight into the functional links among the traits of invaders, residents and the environment that may provide for or diminish opportunities for successful invasion. These features should make our results helpful to informing empirical analyses of the relationship between environmental variation and species invasions.

\section{REFERENCES}

Alpert, P., Bone, E. \& Holzapfel, C. 2000 Invasiveness, invasibility and the role of environmental stress in the spread of non-native plants. Perspect. Plant Ecol. Evol. Syst. 3, 52-66. (doi:10.1078/1433-8319-00004)

Burke, M. J. W. \& Grime, J. P. 1996 An experimental study of plant community invasibility. Ecology 77, 776-790. (doi:10.2307/2265501)

Chesson, P. $2000 a$ Mechanisms of maintenance of species diversity. Annu. Rev. Ecol. Syst. 21, 242-366.

Chesson, P. $2000 b$ General theory of competitive coexistence in spatially-varying environments. Theor. Popul. Biol. 58, 211-237. (doi:10.1006/tpbi.2000.1486)

Condit, R., Aguilar, S., Hernandez, A., Perez, R., Lao, S., Angehr, G., Hubbell, S. P. \& Foster, R. B. 2004 Tropical forest dynamics across a rainfall gradient and the impact of an El Niño fry season. F. Trop. Ecol. 20, 51-72. (doi:10. 1017/S0266467403001081)

Crawley, M. J. 1987 What makes a community invasible? In Colonization, succession, and stability (eds M. J. Crawley, P. J. Edwards \& A. J. Gray), pp. 429-454. Oxford, UK: Blackwell Scientific.

Crawley, M. J., Brown, S. L., Heard, M. S. \& Edwards, G. E. 1999 Invasion-resistance in experimental grassland communities: species richness or species diversity. Ecol. Lett. 2, 140-148. (doi:10.1046/j.1461-0248.1999.00056.x)

Cuddington, K. M. \& Yodzis, P. 1999 Black noise and population persistence. Proc. R. Soc. B 266, 969-973. (doi:10.1098/rspb.1999.0731)

Cyr, H. \& Cyr, I. 2003 Temporal scaling of temperature variability from land to oceans. Evol. Ecol. Res. 5, 1183-1197.

D’Antonio, C. M. \& Levine, J. M. 1999 Elton revisited: a review of evidence linking diversity and invasibility. Oikos 87, 15-26. (doi:10.2307/3546992)

Davis, M. A., Grime, J. P. \& Thompson, K. 2000 Fluctuating resources in plant communities: a general theory of invasibility. F. Ecol. 88, 528-536. (doi:10.1046/j.13652745.2000.00473.x)

Davis, M. A. \& Pelsor, M. 2001 Experimental support for a resource-based mechanistic model of invasibility. Ecol. Lett. 4, 421-428. (doi:10.1046/j.1461-0248.2001.00246.x)

Davis, M. A., Thompson, K. \& Grime, J. P. 2005 Invasibility: the local mechanism driving community assembly and species diversity. Ecography 28, 696-704. (doi:10.1111/ j.2005.0906-7590.04205.x)

Edelstein-Keshet, L. 2005 Mathematical models in biology. Philadelphia, PA: Society for Industrial and Applied Mathematics.

Foster, B. L. \& Dickson, T. L. 2004 Grassland diversity and productivity: the interplay of resource availability and propagule pools. Ecology 85, 1541-1547.

Gilbert, B. \& Lechowicz, M. J. 2005 Invasibility and abiotic gradients: the positive correlation between native and exotic plant diversity. Ecology 87, 1848-1855.

Goldberg, D. E., Rajaniemi, T., Gurevitch, J. \& StewartOaten, A. 1999 Empirical approaches to quantifying interaction intensity: competition and facilitation along productivity gradients. Ecology 80, 1118-1131. (doi:10. 2307/177059)

Huston, M. A. 1994 Biological diversity. Cambridge, UK: Cambridge University Press. 
Huston, M. A. 2004 Management strategies for plant invasions: manipulating productivity, disturbance, and competition. Divers. Distrib. 10, 167-178. (doi:10.1111/ j.1366-9516.2004.00083.x)

Kaitala, V., Ylikarjula, J., Ranta, E. \& Lundperg, P. 1997 Population dynamics and the colour of environmental noise. Proc. R. Soc. B 264, 943-948. (doi:10.1098/rspb. 1997.0130)

Leibold, M. A. et al. 2004 The metacommunity concept: a framework of multi-scale community ecology. Ecol. Lett. 7, 601-613. (doi:10.1111/j.1461-0248.2004.00608.x)

Levine, J. M. \& Rees, M. 2004 Effects of temporal variability on rare plant persistence in annual systems. Am. Nat. 164, 350-363. (doi:10.1086/422859)

Lodge, D. M. 1993 Biological invasions: lessons from ecology. Trends Ecol. Evol. 8, 133-136. (doi:10.1016/ 0169-5347(93)90025-K)

Lonsdale, W. M. 1999 Global Patterns of plant invasions and the concept of invasibility. Ecology 80, 1522-1536. (doi:10. 2307/176544)

Nisbet, R. M. \& Gurney, W. S. C. 1982 Modelling fluctuating populations. New York, NY: Wiley.

Phillips, O. L. et al. 2004 Pattern and process in Amazon tree turnover, 1976-2001. Phil. Trans. R. Soc. B 359, 381-407. (doi:10.1098/rstb.2003.1438)

Rejmanek, M. \& Richardson, D. M. 1996 What attributes make some plant species more invasive? Ecology 77, 1655-1660. (doi:10.2307/2265768)

Ripa, J., Lundberg, P. \& Kaitala, V. 1998 A general theory of environmental noise in ecological food webs. Am. Nat. 151, 256-263. (doi:10.1086/286116)
Roxburgh, S. H., Shea, K. \& Wilson, J. B. 2004 Intermediate disturbance hypothesis: patch dynamics and mechanisms of species coexistence. Ecology 85, 359-371.

Shea, K. \& Chesson, P. 2002 Community ecology theory as a framework for biological invasions. Trends Ecol. Evol. 17, 170-176. (doi:10.1016/S0169-5347(02)02495-3)

Sher, A. A. \& Hyatt, L. A. 1999 The disturbed resource-flux invasion matrix: a new framework for patterns of plant invasion. Biol. Invasions 1, 107-114. (doi:10.1023/ A:1010050420466)

Snyder, R. E. \& Chesson, P. 2004 How the scales of dispersal, competition, and environmental heterogeneity interact of affect coexistence. Am. Nat. 164, 633-650. (doi:10.1086/ 424969)

Stephenson, N. L. \& van Mantgem, P. J. 2005 Forest turnover rates follow global and regional patterns of productivity. Ecol. Lett. 8, 524-531. (doi:10.1111/j.14610248.2005.00746.x)

Stohlgren, T. J. et al. 1999 Exotic plant species invade hot spots of native plant diversity. Ecol. Monogr. 69, 25-46. (doi:10.2307/2657193)

Tilman, D. 1997 Community invasibility, recruitment limitation,, grassland biodiversity. Ecology 78, 81-92. (doi:10.2307/2265980)

Vasseur, D. A. \& Yodzis, P. 2004 The color of environmental noise. Ecology 85, 1146-1152.

Walker, S., Wilson, J. B. \& Lee, W. G. 2005 Does fluctuating resource availability increase invasibility? Evidence from field experiments in New Zealand short tussock grassland. Biol. Inv. 7, 195-211. (doi:10.1007/s10530-0048976-7) 